\title{
Correction to: Some Results on L-Functions Related to Sharing Two Finite Sets
}

\author{
Pulak Sahoo $^{1} \cdot$ Samar Halder $^{1}$
}

Received: 10 December 2020 / Revised: 31 August 2021 / Accepted: 1 September 2021 /

Published online: 3 December 2021

c Springer-Verlag GmbH Germany, part of Springer Nature 2021

\section{Correction to:}

\section{Computational Methods and Function Theory (2019) 19:601-612 https://doi.org/10.1007/s40315-019-00281-0}

In what follows, by Theorem 1 (resp. Theorem 2) we mean Theorem 1 (resp. Theorem 2) of [4]. In the proof of Theorem 2 (see [4, p. 608, before (3.3)]) the argument that "Since $f$ and $\mathcal{L}$ share the set $S_{2}=\left\{b_{1}, b_{2}\right\} \mathrm{CM}$, it follows that $P$ and $Q$ share the set $\left\{\omega_{1}, \omega_{2}\right\}$ CM..." is not true in general. The proof is therefore incorrect as it depends on the said argument. A modification on the values of $m$ related to the elements of $S_{1}$ is required to correct the entire proof, and so the same is needed in the statement of Theorem 2. Moreover, in the rectified statement, IM-sharing instead of CM-sharing of the set $S_{2}$ becomes sufficient to get the conclusion.

Before giving the corrected statement and the proof of Theorem 2, we introduce the following functions regarding the set $S_{1}=\left\{a_{1}, a_{2}, \ldots, a_{m}\right\} \subset \mathbb{C}$, in view of the fact that either $0 \notin S_{1}$ or $0 \in S_{1}$. Let

$$
R_{1}(z)=\frac{z^{m}-\left(\sum a_{i}\right) z^{m-1}+\cdots+(-1)^{m-2}\left(\sum a_{i_{1}} a_{i_{2}} \cdots a_{i_{m-2}}\right) z^{2}}{(-1)^{m}\left(\sum a_{i_{1}} a_{i_{2}} \cdots a_{i_{m-1}}\right) z+(-1)^{m+1} a_{1} a_{2} \cdots a_{m}}
$$

Communicated by Doron Lubinsky.

The original article can be found online at https://doi.org/10.1007/s40315-019-00281-0.

\section{Pulak Sahoo}

sahoopulak1@gmail.com

Samar Halder

samarhalder.mtmh@gmail.com

1 Department of Mathematics, University of Kalyani, Kalyani, West Bengal 741235, India 
where $a_{i} \neq 0, i=1,2, \ldots, m$, and

$$
\begin{aligned}
& \left(z-a_{1}\right)\left(z-a_{2}\right) \cdots\left(z-a_{m}\right) \\
& \quad=z^{m}-\left(\sum a_{i}\right) z^{m-1}+\cdots+(-1)^{m} a_{1} a_{2} \cdots a_{m}=P_{1}(z),
\end{aligned}
$$

say.

Also, let

$$
R_{2}(z)=\frac{z^{m-1}-\left(\sum a_{i}\right) z^{m-2}+\cdots+(-1)^{m-3}\left(\sum a_{i_{1}} a_{i_{2}} \cdots a_{i_{m-3}}\right) z^{2}}{(-1)^{m-1}\left(\sum a_{i_{1}} a_{i_{2}} \cdots a_{i_{m-2}}\right) z+(-1)^{m} a_{1} a_{2} \cdots a_{m-1}},
$$

where $a_{i} \neq 0, i=1,2, \ldots, m-1$, and

$$
\begin{aligned}
& z\left(z-a_{1}\right)\left(z-a_{2}\right) \cdots\left(z-a_{m-1}\right) \\
& \quad=z\left[z^{m-1}-\left(\sum a_{i}\right) z^{m-2}+\cdots+(-1)^{m-1} a_{1} a_{2} \cdots a_{m-1}\right]=P_{2}(z),
\end{aligned}
$$

say.

Suppose that the numerator and the denominator of each of $R_{1}(z)$ and $R_{2}(z)$ have no common zero. Otherwise, we redefine them by adding one more term in the numerator in the same way such that the denominator contains only the non-zero constant term.

Let the number of simple zeros and multiple zeros of the numerator of $R_{1}(z)$ be $\mu_{1}$ and $v_{1}$ respectively, where $\mu_{1}+v_{1} \geq 2$. Also, let the number of simple zeros and multiple zeros of the numerator of $z R_{2}(z)-z+1$ be $\mu_{2}$ and $\nu_{2}$ respectively, where $\mu_{2}+v_{2} \geq 2$.

With the above defined $\mu_{i}$ and $v_{i}(i=1,2)$, the corrected statement of Theorem 2 is given as follows:

Theorem 2 Let $f$ be a meromorphic function in $\mathbb{C}$ with finitely many poles, $m$ be a positive integer. Suppose that $S_{1}=\left\{a_{1}, a_{2}, \ldots, a_{m}\right\}, S_{2}=\left\{b_{1}, b_{2}\right\}$ be two subsets of $\mathbb{C}$ such that $S_{1} \cap S_{2}=\varnothing$ and $\left(b_{1}-a_{1}\right)^{2}\left(b_{1}-a_{2}\right)^{2} \cdots\left(b_{1}-a_{m}\right)^{2} \neq\left(b_{2}-a_{1}\right)^{2}\left(b_{2}-\right.$ $\left.a_{2}\right)^{2} \cdots\left(b_{2}-a_{m}\right)^{2}$. If $f$ and a non-constant $L$-function $\mathcal{L}$ share $S_{1}$ IM and $S_{2} I M$, and one of these conditions is satisfied:

(i) $m>2 \mu_{1}+4 v_{1}+5$ when $S_{1}$ has the form $S_{1}=\left\{a_{1}, a_{2}, \ldots, a_{m}\right\}, a_{i} \neq 0$, $i=1,2, \ldots, m$,

(ii) $m>2 \mu_{2}+4 v_{2}+5$ when $S_{1}$ has the form $S_{1}=\left\{a_{1}, a_{2}, \ldots, a_{m-1}, 0\right\}$, then $\mathcal{L}=f$.

The examples (Example 2 and Example 3) as mentioned in [4, Rem. 1] should be applicable to Theorem 1 only. The following remark is related to Theorem 2.

Remark 2 Considering $S_{1}=\left\{z: z\left[(z+1)^{11}+1\right]=0\right\}=\left\{a_{1}, a_{2}, \ldots, a_{12}\right\}, S_{2}=$ $\{-1,1\}$, we see that $S_{1} \cap S_{2}=\varnothing$ and $\prod_{i=1}^{12}\left(-1-a_{i}\right)^{2} \neq \prod_{i=1}^{12}\left(1-a_{i}\right)^{2}$. This suggests that such sets $S_{1}, S_{2}$ do exist, and the conclusion of Theorem 2 holds if $f$ and $\mathcal{L}$ share $S_{1}, S_{2}$ IM satisfying the value of $m$. 
In order to prove the rectified version of Theorem 2, we use the symbol $H$ to denote the following function:

$$
H=\left(\frac{F^{\prime \prime}}{F^{\prime}}-\frac{2 F^{\prime}}{F-1}\right)-\left(\frac{G^{\prime \prime}}{G^{\prime}}-\frac{2 G^{\prime}}{G-1}\right),
$$

where $F$ and $G$ are two non-constant meromorphic functions. By $N_{E}^{1)}(r, 1 ; F)$ we shall mean the counting function of common simple 1-points of $F$ and $G$, and by $\bar{N}(r, 1 ; F \mid \geq 2)$ we shall mean the reduced counting function of those 1-points of $F$ whose multiplicities are not less than 2 .

The additional lemmas required for the proof, and also the corrected proof of Theorem 2 are given below. The following lemma is obtained by combining [5, Lem. 2] and [2, Lem. 3].

Lemma 6 If two non-constant meromorphic functions $F$ and $G$ share 1 IM and $H \not \equiv 0$, then

$$
\begin{aligned}
N_{E}^{1)}(r, 1 ; F) \leq & \bar{N}(r, 0 ; F \mid \geq 2)+\bar{N}(r, 0 ; G \mid \geq 2)+\bar{N}(r, F \mid \geq 2)+\bar{N}(r, G \mid \geq 2) \\
& +\bar{N}_{*}(r, 1 ; F, G)+\bar{N}_{0}\left(r, 0 ; F^{\prime}\right) \\
& +\bar{N}_{0}\left(r, 0 ; G^{\prime}\right)+S(r, F)+S(r, G),
\end{aligned}
$$

where $\bar{N}_{0}\left(r, 0 ; F^{\prime}\right)$ denotes the reduced counting function of those zeros of $F^{\prime}$ which are not the zeros of $F(F-1)$, and $\bar{N}_{*}(r, 1 ; F, G)$ denotes the reduced counting function of those common 1-points of $F$ whose multiplicities differ from the multiplicities of the corresponding 1-points of $G$.

Taking $m=0$ in [1, Lem. 2.2] we obtain the following lemma.

Lemma 7 Let $F$ and $G$ be two non-constant meromorphic functions sharing 1 IM. Then

$$
\begin{aligned}
& \bar{N}(r, 1 ; F)+\bar{N}(r, 1 ; G)-N_{E}^{1)}(r, 1 ; F)-\frac{1}{2} \bar{N}_{*}(r, 1 ; F, G) \\
& \quad \leq \frac{1}{2}[N(r, 1 ; F)+N(r, 1 ; G)]
\end{aligned}
$$

Lemma 8 Let $F_{1}=R_{1}(f), G_{1}=R_{1}(g), F_{2}=f R_{2}(f)-f+1$ and $G_{2}=g R_{2}(g)-$ $g+1$, where $f$ and $g$ are two non-constant meromorphic functions in $\mathbb{C}$. If $H \neq \equiv$ (taking $H$ as a function of $F_{j}, G_{j}$ ), and $F_{j}, G_{j}$ share 1 IM for any $j \in\{1,2\}$, then

$$
\begin{aligned}
\frac{m}{2}[T(r, f)+T(r, g)] \leq & N_{2}\left(r, 0 ; F_{j}\right)+N_{2}\left(r, 0 ; G_{j}\right)+N_{2}\left(r, F_{j}\right)+N_{2}\left(r, G_{j}\right) \\
& +\frac{3}{2}[N(r, 0 ; f)+N(r, 0 ; g)+\bar{N}(r, f)+\bar{N}(r, g)] \\
& +S(r, f)+S(r, g),
\end{aligned}
$$

where $N_{2}(r, a ; f)=\bar{N}(r, a ; f)+\bar{N}(r, a ; f \mid \geq 2)$ for any $a \in \mathbb{C} \cup\{\infty\}$. 
Proof We shall prove the lemma only for $j=2$, because the other case can be proved in the same manner. Using Nevanlinna's second fundamental theorem, the ValironMokhonko lemma (see [3]), and Lemmas 6 and 7, we have

$$
\begin{aligned}
m[ & T(r, f)+T(r, g)] \\
= & T\left(r, F_{2}\right)+T\left(r, G_{2}\right) \\
\leq & \bar{N}\left(r, 1 ; F_{2}\right)+\bar{N}\left(r, 1 ; G_{2}\right)+\bar{N}\left(r, 0 ; F_{2}\right)+\bar{N}\left(r, 0 ; G_{2}\right)+\bar{N}\left(r, F_{2}\right) \\
& +\bar{N}\left(r, G_{2}\right)-\bar{N}_{0}\left(r, 0 ; F_{2}^{\prime}\right)-\bar{N}_{0}\left(r, 0 ; G_{2}^{\prime}\right)+S\left(r, F_{2}\right)+S\left(r, G_{2}\right) \\
\leq & \frac{1}{2}\left[N\left(r, 1 ; F_{2}\right)+N\left(r, 1 ; G_{2}\right)\right]+\frac{3}{2} \bar{N}_{*}\left(r, 1 ; F_{2}, G_{2}\right)+N_{2}\left(r, 0 ; F_{2}\right) \\
& +N_{2}\left(r, 0 ; G_{2}\right)+N_{2}\left(r, F_{2}\right)+N_{2}\left(r, G_{2}\right)+S\left(r, F_{2}\right)+S\left(r, G_{2}\right) .
\end{aligned}
$$

Let $\bar{N}_{L}\left(r, 1 ; F_{2}\right)$ (resp. $\left.\bar{N}_{L}\left(r, 1 ; G_{2}\right)\right)$ denote the reduced counting function of those common 1-points of $F_{2}, G_{2}$, where the multiplicity of the 1-point of $F_{2}$ (resp. $G_{2}$ ) is greater than that of $G_{2}\left(\right.$ resp. $\left.F_{2}\right)$. Then $\bar{N}_{*}\left(r, 1 ; F_{2}, G_{2}\right)=\bar{N}_{L}\left(r, 1 ; F_{2}\right)+$ $\bar{N}_{L}\left(r, 1 ; G_{2}\right)$.

We note that a 1-point of $F_{2}$ comes only from a zero of $P_{2}(f)$, and $P_{2}(z)$ has all simple zeros. Using Nevanlinna's first fundamental theorem we obtain

$$
\begin{aligned}
\bar{N}_{L}\left(r, 1 ; F_{2}\right) & \leq \bar{N}\left(r, 1 ; F_{2} \mid \geq 2\right) \\
& \leq \bar{N}\left(r, 0 ; P_{2}(f) \mid \geq 2\right) \\
& \leq N\left(r, 0 ; P_{2}(f)\right)-\bar{N}\left(r, 0 ; P_{2}(f)\right) \\
& \leq \sum_{i=1}^{m-1}\left(N\left(r, a_{i} ; f\right)-\bar{N}\left(r, a_{i} ; f\right)\right)+N(r, 0 ; f)-\bar{N}(r, 0 ; f) \\
& \leq N\left(r, 0 ; \frac{f^{\prime}}{f}\right)-N_{\otimes}\left(r, 0 ; f^{\prime}\right)+N(r, 0 ; f)-\bar{N}(r, 0 ; f) \\
& \leq \bar{N}(r, f)+N(r, 0 ; f)-N_{\otimes}\left(r, 0 ; f^{\prime}\right)+S(r, f)
\end{aligned}
$$

where $N_{\otimes}\left(r, 0 ; f^{\prime}\right)=\bar{N}\left(r, 0 ; f^{\prime} \mid f \neq 0 ; a_{1}, a_{2}, \ldots, a_{m-1}\right)$. Similarly, we get

$$
\bar{N}_{L}\left(r, 1 ; G_{2}\right) \leq \bar{N}(r, g)+N(r, 0 ; g)-N_{\otimes}\left(r, 0 ; g^{\prime}\right)+S(r, g) .
$$

This implies

$$
\begin{aligned}
& \frac{m}{2}[T(r, f)+T(r, g)] \\
& \quad \leq \frac{3}{2}[N(r, 0 ; f)+N(r, 0 ; g)+\bar{N}(r, f)+\bar{N}(r, g)]+N_{2}\left(r, 0 ; F_{2}\right) \\
& \quad+N_{2}\left(r, 0 ; G_{2}\right)+N_{2}\left(r, F_{2}\right)+N_{2}\left(r, G_{2}\right)+S(r, f)+S(r, g) .
\end{aligned}
$$

This completes the proof of the lemma. 
Proof of Theorem 2 Let $F_{1}=R_{1}(f), G_{1}=R_{1}(\mathcal{L}), F_{2}=f R_{2}(f)-f+1$ and $G_{2}=\mathcal{L} R_{2}(\mathcal{L})-\mathcal{L}+1$. By [4, Lem. 3], it is obvious that $S(r, f)=S(r, \mathcal{L})=O(\log r)$. Again, we have $\bar{N}(r, f)=\bar{N}(r, \mathcal{L})=O(\log r)$ as $f$ has finitely many poles, and $\mathcal{L}$ has at most one possible pole.

We first assume that $S_{1}$ is of the form: $S_{1}=\left\{a_{1}, a_{2}, \ldots, a_{m}\right\}, a_{i} \neq 0, i=$ $1,2, \ldots, m$. Since $f$ and $\mathcal{L}$ share $S_{1}$ IM, therefore $F_{1}$ and $G_{1}$ share 1 IM. For $F_{1}, G_{1}$, we suppose that $H \neq \equiv 0$. If $\alpha_{1}, \alpha_{2}, \ldots, \alpha_{\mu_{1}}$ and $\beta_{1}, \beta_{2}, \ldots, \beta_{\nu_{1}}$ are respectively the distinct simple zeros and multiple zeros of the numerator of $R_{1}(z)$, and if $\gamma$ is the zero of the denominator of $R_{1}(z)$ (when it exists), then by Lemma 8 we obtain

$$
\begin{aligned}
\frac{m}{2}\{ & T(r, f)+T(r, \mathcal{L})\} \\
\leq & \frac{3}{2}\{N(r, 0 ; f)+N(r, 0 ; \mathcal{L})\}+\sum_{i=1}^{\mu_{1}}\left\{N\left(r, \alpha_{i} ; f\right)+N\left(r, \alpha_{i} ; \mathcal{L}\right)\right\} \\
& +2 \sum_{j=1}^{\nu_{1}}\left\{\bar{N}\left(r, \beta_{j} ; f\right)+\bar{N}\left(r, \beta_{j} ; \mathcal{L}\right)\right\}+N(r, \gamma ; f)+N(r, \gamma ; \mathcal{L}) \\
& +O(\log r) \\
\leq & \left(\frac{5}{2}+\mu_{1}+2 v_{1}\right)\{T(r, f)+T(r, \mathcal{L})\}+O(\log r),
\end{aligned}
$$

which is clearly a contradiction as $m>2 \mu_{1}+4 v_{1}+5$. Therefore our assumption was wrong, and so $H \equiv 0$, which on integration gives $1 /\left(F_{1}-1\right)=c_{1} /\left(G_{1}-1\right)+c_{2}$, where $c_{1}(\neq 0), c_{2}$ are constants. Therefore, $F_{1}$ and $G_{1}$ share $1 \mathrm{CM}$.

Next, we assume that $S_{1}$ is of the form: $S_{1}=\left\{a_{1}, a_{2}, \ldots, a_{m-1}, 0\right\}, a_{i} \neq 0$, $i=1,2, \ldots, m-1$. Clearly, $F_{2}$ and $G_{2}$ share $1 \mathrm{IM}$ as $f$ and $\mathcal{L}$ share $S_{1}$ IM. For $F_{2}, G_{2}$, we suppose that $H \not \equiv 0$. Then, considering the zeros of the numerator and the denominator of $z R_{2}(z)-z+1$ and applying Lemma 8 similarly as above, we get

$$
\frac{m}{2}\{T(r, f)+T(r, \mathcal{L})\} \leq\left(\frac{5}{2}+\mu_{2}+2 v_{2}\right)\{T(r, f)+T(r, \mathcal{L})\}+O(\log r),
$$

a contradiction as $m>2 \mu_{2}+4 \nu_{2}+5$. Therefore the assumption that $H \not \equiv 0$ is also proved wrong for the functions $F_{2}, G_{2}$, and so $H \equiv 0$, which implies, $F_{2}$ and $G_{2}$ share $1 \mathrm{CM}$.

Therefore, for $m>2 \mu_{j}+4 v_{j}+5$, and for any $j \in\{1,2\}$, we obtain that $F_{j}$ and $G_{j}$ share $1 \mathrm{CM}$.

For a particular $j \in\{1,2\}$, since zeros of $F_{j}-1$ and $G_{j}-1$ are the zeros of $P_{j}(f)$ and $P_{j}(\mathcal{L})$ respectively, therefore $P_{j}(f)$ and $P_{j}(\mathcal{L})$ share $0 \mathrm{CM}$ whenever $m>2 \mu_{j}+4 v_{j}+5$. Combining this with the fact that $f$ and $\mathcal{L}$ share $S_{1}$ IM, and that $P_{j}(z)$ has only simple zeros, we conclude that $f$ and $\mathcal{L}$ share $S_{1} \mathrm{CM}$. Then, by Theorem 1 we obtain the conclusion of this theorem. This completes the proof.

In $[4$, Lem. 1], “CC" should be replaced by " $\mathbb{R} "$. 


\section{References}

1. Banerjee, A.: Uniqueness of meromorphic functions sharing two sets with finite weight II. Tamkang J. Math. 41, 379-392 (2010)

2. Lahiri, I.: Weighted value sharing and uniqueness of meromorphic functions. Complex Var. Theory Appl. 46, 241-253 (2001)

3. Mokhon'ko, A.Z.: On the Nevanlinna characteristics of some meromorphic functions, theory of functions. Funct. Anal. Appl. 14, 83-87 (1971)

4. Sahoo, P., Halder, S.: Some results on L-functions related to sharing two finite sets. Comput. Methods Funct. Theory 19, 601-612 (2019)

5. Yi, H.X.: Meromorphic functions that share one or two values II. Kodai Math. J. 22, 264-272 (1999)

Publisher's Note Springer Nature remains neutral with regard to jurisdictional claims in published maps and institutional affiliations. 